\title{
CELIAC DISEASE OR GLUTEN RELATED DISORDERS? WHO WOULD BE THE DISEASE OF THE TIME?
}

RLS Soares. Celiac disease or Gluten Related Disorders? Who would be the disease of the time? Arq Gastroenterol. 2015,52(3):165-6.

In recent years it has been observed the significant increase in the number of scientific publications regarding Celiac Disease (CD) and other Gluten Related Disorders (GRDs). Until two decades ago, CD was believed to be unusual or even rare outside of Europe and was relatively ignored by health professionals and the global media. Today CD can be considered a global health problem, affecting of $0.6 \%$ to $1.0 \%$ of the population in general, including populations of Latin America and the Middle East ${ }^{(2,6)}$. The estimated prevalence of $\mathrm{CD}$ in Latin America ranges from $0.46 \%$ to $0.64 \%$, frequency similar to European countries, including Brazil ${ }^{(3,4)}$. This increased prevalence of CD appears to be associated with the two sets of predominant factors.

The first set would be related to discovery of important milestones and pathogenic diagnoses in recent years that led CD from obscurity to global prominence. Serological tests are used for screening and histological abnormalities confirmed the diagnosis, and the only known treatment is a gluten-free diet for long periods. However, there are several notable issues and unresolved questions in the epidemiology of CD that include the role of benefits and drawbacks of screening high-risk populations for $\mathrm{CD}$, the clinical significance of $\mathrm{CD}$ detected by mass screening and the prevalence of undiagnosed $\mathrm{CD}$, and what are the most appropriate $\mathrm{CD}$ specific quality of life measures. It also remains to be determined whether the higher incidence of $\mathrm{CD}$ is due to a true increase or simply reflects increased testing for $\mathrm{CD}$.

The second set of data associated with the increased prevalence of $\mathrm{CD}$ and GRDs could be related to an increase in the global consumption of wheat in recent decades. Cereals that contain gluten are widely consumed and current wheat varieties have a higher content in gluten compared to the past due to changes directed by both technology and nutritional reasons. On the other hand, the increased amount of gluten in wheat's current composition and possible associated symptoms lead people to avoid products that contain wheat and gluten. It is also a global phenomenon.

These two sets of associated factors led to a broadening of the spectrum of clinical definition of gluten intolerance in the last twenty years. In the 1980s, classification of GRDs was very simple, because CD and Dermatitis Herpetiformis (DH) were the only known diseases with a well-documented role of gluten in their pathogenesis. Increasing complexity in the nomenclature and clinical presentation of GRDs has led to the development of a consensus document by a panel of experts on new classification of five heterogeneous GRDs: CD, Non-Coeliac Gluten Sensitivity (NCGS), Wheat Allergy (WA) DH, and gluten ataxia (GA) although there can show considerable overlap in the clinical presentation $^{(1,6)}$.

The most famous of the GRDS is NCGS. It's a condition where intestinal and extra-intestinal symptoms are triggered by gluten ingestion in the absence of CD and WA. The clinical picture of NCGS is a combination of IBS-like symptoms, behaviour disturbances and systemic manifestations. Despite the first reports on NCGS dating from 1978, much remains unknown about the condition ${ }^{(1,2,6)}$. The increasing prevalence and complexity of GRDs inevitably has stimulated increasing interest in gluten-free diets in scientific and non-scientific communities. However, while CD is well established, many questions remain about the possibility of gluten be a trigger of gastrointestinal and or extraintestinal symptoms in patients without CD. NCGS exist? There is some evidence that NCGS may exist, but probably only in a small number of people. In contrast to CD, the self reported NCGS patients are heterogeneous and are highly suggestible, making a largely difficult patient group to study. Thus efficient diagnostic criteria are needed to distinguish GFD people with a medical condition from those 
who simply prefer to avoid gluten, leading to different nutrition and monitoring strategies. How it induces systemic symptoms? One speculative hypothesis is that gluten may not be directly involved in the triggering of GI symptoms, but rather in the pathogenesis of visceral hypersensitivity, like IBS. Aside from the well defined medical condition of $\mathrm{CD}$, gluten is blamed as a trigger of symptoms by $20 \%-45 \%$ of adults who self-report food hypersensitivity ${ }^{(2)}$. However, the role of dietary components in inducing gastrointestinal symptoms is a complex area and the understanding of dietary triggers of GI symptoms is not complete ${ }^{(5)}$. In relation to the treatment of NCGS many questions remain unanswered and it needs to be verified whether the elimination of dietary gluten alone is sufficient for the control of symptoms, and to understand the overlap with other components of wheat. However, unnecessarily following restrictive diets raise two main concerns. Firstly, the prescription of a gluten-free diet for gastrointestinal and other symptoms may lead to the under diagnosis of $\mathrm{CD}$. Secondly, long-term restrictive diets, particularly avoidance of wheat-based products, are likely to have health implications especially given their important role in bowel health .In conclusion, the thin line between $\mathrm{CD}$, WA, irritable bowel syndrome and NCGS is not always clearly distinguishable. Providing the careful design of clinical trials, the next several years will provide a stronger quality of evidence.

Finally, we believe that the recognition of $\mathrm{CD}$ as a global problem, the need for better prevention of the development of the disease and its complications, as well as the need for better clinical understanding of the nature of NCGS including your trigger, diagnosis, treatment and risks, justify the recent increase in gluten-related diseases research.

In this issue are published three original articles related to CD: "The Neurological Face of Celiac Disease"; "Quality of Life Evaluation in Celiac Patients from Southern Brazil" and "Low Bone Mineral Density in Brazilian Patients at Diagnosis of Celiac Disease".

Enjoy!

RLS Soares. Doenças relacionadas ao glúten - Quem são os novos personagens? Arq Gastroenterol. 2015,52(3):165-6.

\section{REFERENCES}

1. Biesiekierski JR, Iven J. Non-coeliac gluten sensitivity: piecing the puzzle together United European Gastroenterology Journal. 2015;3(2):160-5.

2. Green PH, Lebwohl B, Greywoode R. Celiac disease. J Allergy Clin Immunol. 2015;135(5):1099-106.

3. Kotze, L MS. Celiac disease in Brazilian patients: associations, complications and causes of death. Forty years of clinical experience. Arq. Gastroenterol. 2009;46(4):261-9.
4. Parra-Medina R, Molano-Gonzalez N, Rojas-Villarraga A, Agmon-Levin N, Arango M-T, Shoenfeld Y, et al. (2015) Prevalence of Celiac Disease in Latin America: A Systematic Review and Meta-Regression. PLoS ONE 10(5):e0124040.

5. Soares RL, Figueiredo HN, Santos JM, Oliveira RF, Godoy RL, Mendonça FA. Discrepancies between the responses to skin prick test to food and respiratory antigens in two subtypes of patients with irritable bowel syndrome. World Journal of Gastroenterology: WJG. 2008;14(19):3044-8

6. Tovoli F, Masi C, Guidetti E, Negrini G, Paterini P, Bolondi L. Clinical and diagnostic aspects of gluten related disorders. World Journal of Clinical Cases: WJCC. 2015;3(3):275-84 